EPJ manuscript No.

(will be inserted by the editor)

\title{
Magnetic moment of an electron gas on the surface of constant negative curvature
}

\author{
D.V. Bulaev and V.A. Margulis \\ Mordovian State University, Saransk, 430000 Russia \\ Received: date / Revised version: date
}

\begin{abstract}
The magnetic moment of an electron gas on the surface of constant negative curvature is investigated. It is shown that the surface curvature leads to the appearance of the region of the monotonic dependence $M(B)$ at low magnetic fields. At high magnetic fields, the dependence of the magnetic moment on a magnetic field is the oscillating one. The effect of the surface curvature is to increase the region of the monotonic dependence of the magnetic moment and to break the periodicity of oscillations of the magnetic moment as a function of an inverse magnetic field.
\end{abstract}

PACS. 73.20.At Surface states, band structure, electron density of states $-75.75 .+$ a Magnetic properties of nanostructures

\section{Introduction}

The two-dimensional electron gas (2DEG) in quantized magnetic fields has attracted a lot of attention in recent years. The increasing interest to the 2DEG is due to its unique properties: periodic oscillations of the magnetic moment as a function of an inverse magnetic field (the de Haas - van Alphen effect), oscillations of the longitudinal magnetoresistance (the Shubnikov - de Haas effect), ' and quantization of the Hall conductivity (the integer and fractional quantum Hall effects). Transport and magnetic measurements are powerful methods for studying the electron energy spectrum. Note that transport measurements yield information about the localized states near the Fermi ' energy, but provide very little direct information about the total density of states of the 2DEG. In contrast, measurements of magnetization are a powerful tool for exploring the total density of states of a degenerate Fermi gas. Recent advances in magnetometry provide new insights into the electron density of states of the 2DEG [1].

The theory of the two-dimensional de Haas - van Alphen effect was started by Peierls 2. In the zero-temperature limit he obtained that the magnetization in the ideal 2DEG has sharp, saw-tooth oscillations with a constant amplitude. Recent theoretical investigations show that in the two-dimensional case the dependence of the oscillation amplitude on a magnetic field and temperature differs from the Lifshitz - Kosevich formula for bulk conductors [3], and this is confirmed by experiments [1].

The magnetic properties of electrons on curved surfaces have attracted a substantial interest in recent years. This is due to the fact that these structures have unusual

Correspondence to: bulaevdv@mrsu.ru properties of the magnetic moment as a function of a magnetic field, temperature, and geometric parameters [4,5, 6. 7.8. 8 .

In this paper we study the magnetic moment of electrons on the surface of constant negative curvature (the Lobachevsky plane). Although the Lobachevsky plane has not been experimentally realized yet, the problem of the physics on the Lobachevsky plane has a deep relation with some interesting problems, like the occurrence of the chaos in the surface of negative curvature 9, the Berry phase [10, and point perturbations [11] on the Lobachevsky plane. In recent years, the quantum Hall effect on the Lobachevsky plane is a subject of current interest [12,13. 14,15].

\section{Electron states and magnetic moment}

We consider noninteracting electrons confined to the surface of constant negative curvature (the Lobachevsky plane) in a magnetic field $\mathbf{B}$. We choose the Landau gauge for the vector potential $\left(\mathbf{A}=\left(B a^{2} y^{-1}, 0\right)\right)$ and employ the Poincaré realization in which the Lobachevsky plane $L$ is identified with the upper complex halfplane $L=\{z=$ $x+i y \in \mathbb{C}: y>0\}$ endowed with the metric

$$
d s^{2}=\frac{a^{2}}{y^{2}}\left(d x^{2}+d y^{2}\right),
$$

where $a$ is the radius of curvature. The Hamiltonian of the system can be written as 15

$$
H=\frac{\hbar^{2}}{2 m^{*} a^{2}}\left[-y^{2}\left(\partial_{x}^{2}+\partial_{y}^{2}\right)+2 i b y \partial_{x}+b^{2}-\frac{1}{4}\right],
$$


where $m^{*}$ is the effective electron mass, $b=e B a^{2} / \hbar c$, and the last term in Eq. (10) is the surface potential which arises from the surface curvature [16]. The spectrum of $H$ consists of two parts [17: a discrete spectrum in the interval $\left(0, \hbar^{2} b^{2} / 2 m^{*} a^{2}\right)$ consisting of a finite number of Landau levels

$E_{n}=\hbar \omega_{c}\left(n+\frac{1}{2}\right)-\frac{\hbar^{2}}{2 m^{*} a^{2}}\left(n+\frac{1}{2}\right)^{2}, 0 \leq n<|b|-\frac{1}{2}$

and a continuous spectrum in the interval $\left[\hbar^{2} b^{2} / 2 m^{*} a^{2}, \infty\right)$

$$
E(\nu)=\frac{\hbar^{2}}{2 m^{*} a^{2}}\left(b^{2}+\nu^{2}\right), 0 \leq \nu<\infty .
$$

The electron density of states $n(E)$ per unit area is defined by the following expression:

$$
n(E)=\frac{1}{\pi S} \int \operatorname{Im} G(\mathbf{r}, \mathbf{r} ; E+i 0) d \mathbf{r}
$$

where $S$ is the area of the surface and $G\left(\mathbf{r}, \mathbf{r}^{\prime} ; E\right)$ is the Green's function of the Hamiltonian. The density of states of electrons on the Lobachevsky plane is given by [15]

$$
\begin{aligned}
& n(E)=\frac{1}{2 \pi a^{2}} \sum_{0 \leq n<|b|-1 / 2}\left(|b|-n-\frac{1}{2}\right) \delta\left(E-E_{n}\right) \\
& +\frac{m^{*}}{2 \pi \hbar^{2}} \Theta\left(E-\frac{\hbar^{2} b^{2}}{2 m^{*} a^{2}}\right) \\
& \times \frac{\sinh 2 \pi \sqrt{2 m^{*} a^{2} E / \hbar^{2}-b^{2}}}{\cosh 2 \pi \sqrt{2 m^{*} a^{2} E / \hbar^{2}-b^{2}}+\cos 2 \pi b}
\end{aligned}
$$

where $\Theta(x)$ is the Heaviside step function. The first term in Eq. (3) corresponds to the discrete spectrum and the second term corresponds to the continuous one and coincides with the expression given in Ref. [17.

The magnetic moment of a thermodynamic system with a fixed chemical potential is given by

$$
\mathbf{M}=-\left.\left(\frac{\partial \Omega}{\partial \mathbf{B}}\right)\right|_{T, S, \mu},
$$

where

$$
\Omega(T, \mu)=-T S \int_{-\infty}^{\infty} n(E) \ln \{1+\exp [(\mu-E) / T]\} d E
$$

is the thermodynamic potential.

First, we consider the case of zero temperature. We substitute the formula for the electron density of states (Eq. (3) into Eq. (5) and apply the Poisson summation formula for the magnetic moment. When both the discrete spectrum and the continuous one are below the Fermi energy, i.e, when $\varepsilon_{F} \geq b^{2}\left(\varepsilon_{F}=2 m^{*} a^{2} E_{F} / \hbar^{2}\right)$, the magnetic moment of an $2 \mathrm{DEG}$ on the Lobachevsky plane is given by

$$
\left.\frac{M(T=0)}{\mu_{B}}\right|_{\varepsilon_{F} \geq b^{2}}=\frac{m_{e}}{m^{*}} \frac{S}{2 \pi a^{2}}\left\{-\frac{b}{6}\right.
$$

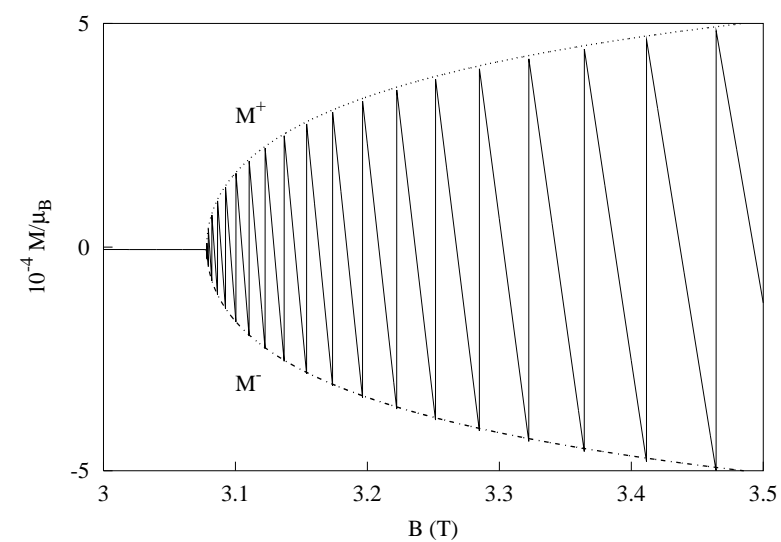

Fig. 1. The magnetic moment of a $2 \mathrm{DEG}$ on the Lobachevsky plane as a function of a magnetic field; $a=10^{-5} \mathrm{~cm}, S=$ $3 \times 10^{-9} \mathrm{~cm}^{2}, E_{F}=2 \times 10^{-13} \mathrm{erg}$.

$$
\begin{aligned}
& -\frac{3}{2 \pi^{3}} \sum_{k=1}^{\infty} \frac{(-1)^{k}}{k^{3}} \exp \left[-2 \pi k \sqrt{\varepsilon_{F}-b^{2}}\right] \sin 2 \pi k b \\
& +\frac{2}{\pi} \sqrt{\varepsilon_{F}-b^{2}} \sum_{k=1}^{\infty} \frac{(-1)^{k}}{k} \exp \left[-2 \pi k \sqrt{\varepsilon_{F}-b^{2}}\right] \\
& \times\left(b \cos 2 \pi k b-\sqrt{\varepsilon_{F}-b^{2}} \sin 2 \pi k b\right) \\
& +\frac{1}{\pi^{2}} \sum_{k=1}^{\infty} \frac{(-1)^{k}}{k^{2}} \exp \left[-2 \pi k \sqrt{\varepsilon_{F}-b^{2}}\right] \\
& \left.\times\left(b \cos 2 \pi k b-3 \sqrt{\varepsilon_{F}-b^{2}} \sin 2 \pi k b\right)\right\} .
\end{aligned}
$$

As can be seen from Eq. (6), in the region $\varepsilon_{F} \geq b^{2}, M(B)$ is the monotonic dependence, since the exponents in the sums are negligible.

For $\varepsilon_{F}<b^{2}$ (in this case only the discrete spectrum is below the Fermi energy), the magnetic moment is given by

$$
\begin{aligned}
& \left.\frac{M(T=0)}{\mu_{B}}\right|_{\varepsilon_{F}<b^{2}}=\frac{m_{e}}{m^{*}} \frac{S}{2 \pi a^{2}}\left\{-\frac{b}{6}\right. \\
& -\frac{3}{2 \pi^{3}} \sum_{k=1}^{\infty} \frac{(-1)^{k}}{k^{3}} \sin 2 \pi k\left(b-\sqrt{b^{2}-\varepsilon_{F}}\right) \\
& +\frac{1}{\pi^{2}}\left(b-3 \sqrt{b^{2}-\varepsilon_{F}}\right) \sum_{k=1}^{\infty} \frac{(-1)^{k}}{k^{2}} \cos 2 \pi k\left(b-\sqrt{b^{2}-\varepsilon_{F}}\right) \\
& -\frac{2}{\pi} \sqrt{b^{2}-\varepsilon_{F}}\left(b-\sqrt{b^{2}-\varepsilon_{F}}\right) \\
& \left.\times \sum_{k=1}^{\infty} \frac{(-1)^{k}}{k} \sin 2 \pi k\left(b-\sqrt{b^{2}-\varepsilon_{F}}\right)\right\} .
\end{aligned}
$$

As can be seen from Eq. (7), the dependence $M(B)$ has the monotonic part, which is a linear function of a magnetic field, and the three sums lead to saw-tooth oscillations.

In Fig. 1 we show the dependence $M(B)$. The dotted and dot-dashed lines show the envelope of maxima and minima of the magnetic moment respectively. 
The monotonic dependence $M(B)$ corresponds to the case when both the discrete spectrum and the continuous one are below the Fermi energy. The bottom of the continuous spectrum crosses the Fermi level with increasing a magnetic field, therefore, there is only the discrete spectrum below the Fermi energy. In this case, the monotonic dependence of the magnetic moment on a magnetic field is replaced by the oscillating one. As can be seen from Fig. 11 for $\varepsilon_{F}<b^{2}$, the monotonic part of the magnetic moment is much less than the amplitude of saw-tooth oscillations. The jumps in the magnetic moment arise from the crossings of the Fermi level by the electron levels.

Let us consider the monotonic dependence $M(B)$. As can be seen from Eq. (6), the exponents in the sums are negligible for $\varepsilon_{F} \gg b^{2}$. Neglecting these sums, we have that the dependence of the magnetic moment on a magnetic field is almost linear

$$
\left.\frac{M(T=0)}{\mu_{B}}\right|_{\varepsilon_{F} \gg b^{2}} \simeq \frac{m_{e}}{m^{*}} \frac{S}{2 \pi a^{2}}\left(-\frac{b}{6}\right) .
$$

Now consider the magnetic moment oscillations in the region $\varepsilon_{F}<b^{2}$. From Eq. (17) it is easy to find the envelope of the maxima and minima of the magnetic moment

$$
\frac{M^{ \pm}}{\mu_{B}}=\frac{m_{e}}{m^{*}} \frac{S}{2 \pi a^{2}} \sqrt{b^{2}-\varepsilon_{F}}\left( \pm b-\frac{1}{2} \mp \sqrt{b^{2}-\varepsilon_{F}}\right),
$$

where $M^{+}$and $M^{-}$are the envelopes of the maxima and minima of the magnetic moment respectively. The difference between them is the oscillation amplitude:

$\frac{\Delta M}{\mu_{B}}=\frac{M^{+}-M^{-}}{\mu_{B}}=2 \frac{m_{e}}{m^{*}} \frac{S}{2 \pi a^{2}} \sqrt{b^{2}-\varepsilon_{F}}\left(b-\sqrt{b^{2}-\varepsilon_{F}}\right)$

As can be seen from this equation, the surface curvature decreases the amplitude of oscillations of the magnetic moment. The oscillation amplitude tends to $E_{F} 2 m_{e} S / \pi \hbar^{2}$ with a magnetic field. As shown in Fig. 1 oscillations of the magnetic moment as a function of a magnetic field are not periodic. It is easy to find the distance between two neighboring jumps in units of $1 / B$ :

$$
\Delta\left(\frac{1}{B}\right)=\frac{|e| a^{2}}{\hbar c} \frac{2 \sqrt{b^{2}-\varepsilon_{F}}-1}{b\left(b^{2}+2 b+1-2(b+1) \sqrt{b^{2}-\varepsilon_{F}}\right)} .
$$

As can be seen form this equation, the oscillations of the magnetic moment as a function of an inverse magnetic field $(1 / B)$, in contrast with the case of the flat surface, are not periodic. Note that in the limit of zero curvature the distance between two neighboring jumps is given by

$$
\Delta\left(\frac{1}{B}\right) \underset{a \rightarrow \infty}{\longrightarrow} \frac{\hbar|e|}{m^{*} c E_{F}} .
$$

From an analytical study of the obtained formulas and from a numerical analysis we find that the region of the monotonic dependence of the magnetic moment is increasing, the amplitude and the distance between neighboring jumps of the magnetic moment as a function of a magnetic field are decreasing with increasing surface curvature.
Let us now consider the effect of temperature on the magnetic moment of a 2DEG on the Lobachevsky plane. Substituting Eq. (3) into Eq. (5), we get $M=M_{1}+M_{2}$, where

$$
\begin{aligned}
& \frac{M_{1}}{\mu_{B}}=-\frac{m_{e}}{m^{*}} \frac{S}{2 \pi a^{2}}\left\{\sum_{0 \leq n<b-1 / 2}\left(b-n-\frac{1}{2}\right)(2 n+1) f_{0}\left(E_{n}\right)\right. \\
& \left.-\frac{2 m^{*} a^{2} T}{\hbar^{2}} \sum_{0 \leq n<b-1 / 2} \ln \left\{1+\exp \left[\left(\mu-E_{n}\right) / T\right]\right\}\right\}
\end{aligned}
$$

is the contribution of the discrete spectrum,

$$
\begin{aligned}
& \frac{M_{2}}{\mu_{B}}=-\frac{m_{e}}{m^{*}} \frac{S}{2 \pi a^{2}}\left\{b \int_{0}^{\infty} d t \frac{\sinh 2 \pi \sqrt{t}}{\cosh 2 \pi \sqrt{t}+\cos 2 \pi b}\right. \\
& \times f_{0}\left(\frac{\hbar^{2}}{2 m^{*} a^{2}}\left(t+b^{2}\right)\right) \\
& -\frac{2 m^{*} a^{2} T}{\hbar^{2}} \pi \sin 2 \pi b \int_{0}^{\infty} d t \frac{\sinh 2 \pi \sqrt{t}}{(\cosh 2 \pi \sqrt{t}+\cos 2 \pi b)^{2}} \times \\
& \left.\times \ln \left\{1+\exp \left[\left(\mu-\frac{\hbar^{2}}{2 m^{*} a^{2}}\left(t+b^{2}\right)\right) / T\right]\right\}\right\}
\end{aligned}
$$

is the contribution of the continuous spectrum to the magnetic moment. The numerical study shows that temperature results in smearing of the oscillations maxima and decreasing the oscillation amplitude. The monotonic part of the magnetic moment is nearly independent of temperature.

\section{Conclusions}

The magnetic moment of a 2DEG on the surface of constant negative curvature is investigated. It is shown that when both the discrete spectrum and the continuous one are below the Fermi energy, the dependence of the magnetic moment on a magnetic field is the monotonic one. The bottom of the continuous spectrum crosses the Fermi level with increasing a magnetic field. In this case, the monotonic dependence of the magnetic moment on a magnetic field is replaced by the oscillating one. The effect of the surface curvature is to increase the region of the monotonic dependence of the magnetic moment and to decrease the amplitude and the distance between neighboring jumps of the magnetic moment as a function of a magnetic field.

This work was supported by the INTAS Grant No. 00-257 and the Russian Ministry of Education Grant No. E02-3.4370. One of us (D.V.B.) was supported in part by the Russian Ministry of Education Grant No. E02-2.0-15, and the RFBR Grant No. 03-02-06006-mas.

\section{References}

1. J. Harris et al., Phys. Rev. Lett. 86, 4644 (2001) 
2. R. Peierls, Phys. Z 81, 186 (1933)

3. D. Grigoriev, I.D. Vagner, Pis'ma v ZhETF 69, 139 (1999) [JETP Lett. 69, 156 (1999)]

4. J.H. Kim, I.D. Vagner, B. Sundaram, Phys. Rev. B 46, 9501 (1992)

5. D.N. Aristov, Phys. Rev. B 59, 6368 (1999)

6. D.V. Bulaev, V.A. Geyler, V.A. Margulis, Phys. Rev. B 62, 11517 (2000)

7. L.I. Magarill, A.V. Chaplik, Zh. Eksp. Teor. Fiz. 115, 1478 (1999) [Sov. Phys. JETP 88, 815 (1999)]

8. V.A. Geyler, V.A. Margulis, A.V. Shorokhov, Zh. Eksp. Teor. Fiz. 115, 1450 (1999) [Sov. Phys. JETP 88, 800 (1999)]

9. C. Grosche, J. Phys. A 25, 4573 (1992)

10. S.A. Albeverio, P. Exner, V.A. Geyler, Lett. Math. Phys. 55, 9 (2001)

11. J. Brüning, V.A. Geyler, Teor. Matem. Fiz. 115, 368 (1999) [Theor. Math. Phys. 119, 687 (1999)]

12. R. Iengo, D. Li, Nucl. Phys. B, 413, 735 (1994)

13. J.E. Avron et al., Phys. Rev. Lett. 69, 128 (1992)

14. A. Pnueli, Ann. Phys. 231, 56 (1994)

15. D.V. Bulaev, V.A. Geyler, V.A. Margulis, Phisica B (to be published)

16. N.P. Landsman, Mathematical topics between classical and quantum mechanics (Springer-Verlag, New York, 1998)

17. A. Comtet, Ann. Phys. 173, 185 (1987) 